\title{
Accuracy of Blood Pressure Measurement in the Family Practice Center
}

\section{Lawrence $E$. Kay, $M D$}

Background: Equipment, physiologic, and technique factors can influence the accuracy of blood pressure measurement. The current study was designed to isolate certain technique factors and then assess the accuracy of nursing personnel measurements of blood pressure in three residency family practice centers.

Methods: An experienced registered nurse was trained in applying the American Heart Association recommendations for determining blood pressure by sphygmomanometry; three 1.5-hour practice sessions demonstrated her accuracy. Nine full days were then spent in the family practice centers rechecking as many staff blood pressure readings as possible while controlling for confounding variables.

Results: The following findings were significant: (1) the average absolute differences between control and study nurse systolic and diastolic blood pressure readings were $6.2 \mathrm{mmHg}$ and $4.7 \mathrm{mmHg}$, respectively; (2) a unidirectional error of $3.8 \mathrm{mmHg}$ in the measurement of diastolic blood pressure was found in one center, possibly because less care was taken with afternoon measurements; (3) variability in systolic blood pressure readings was higher in all three centers $( \pm 8.5 \mathrm{mmHg})$ than attained during the training sessions for the control nurse $( \pm 5.8 \mathrm{mmHg}$ ); (4) the average errors attributable to technique factors studied that were potentially correctable by training were only $1.8 \mathrm{mmHg}$ for systolic and $0.7 \mathrm{mmHg}$ for diastolic pressures.

Conclusions: The degree of inaccuracy in ambulatory nursing blood pressure readings attributable to errors in technique is quantified by this study. Training can reduce, but not abolish, this inaccuracy. Careful attention to proper blood pressure measurement technique and such variables as equipment calibration is essential for both nursing and physician observers. Taking multiple blood pressure measurements before making clinical decisions can limit the effect of these inaccuracies. (J Am Board Fam Pract 1998;11:252-8.)

Appropriate diagnosis and optimal treatment of hypertension depends upon accurate blood pressure measurement by nursing and physician personnel. Although the accuracy of this common technique is generally assumed in clinical settings, such indirect measurements are fraught with inconsistency. First, the discrepancies between indirect and direct measurements for the same patients range from 3 to $24.6 \mathrm{mmHg}$, emphasizing that indirect blood pressure measurement is, at best, an approximation of the true value. Second, even assuming that indirect blood pressure measurements truly correlate with direct readings, the medical literature on hypertension clearly documents a remarkable degree of variation in auscul-

Submitted, revised, 24 October 1997.

From the Polyclinic Hospital Family Practice Residency, PinnacleHealth System, Harrisburg, $\mathrm{Pa}$. Address reprint requests to Lawrence E. Kay, MD, PinnacleHealth at Polyclinic Hospital, Kline Building, Room 209, 2601 North Third Street, Harrisburg, Pa. 17110.

Financial support for this project was provided by the PinnacleI Iealth Department of Family Practice. tatory blood pressure values resulting from multiple potentially controllable factors ${ }^{2}$ (Table 1 ).

The American Heart Association (AHA) recommendations for determining blood pressure by sphygmomanometry were developed to standardize blood pressure measurement protocol, thereby controlling for many of these factors and allowing for greater comparability of readings between studies. Although the diligent teaching and implementation of these guidelines have undoubtedly led to more comparable measurements, medical practitioners continue to show lack of consistency in fully adhering to the established protocol. ${ }^{5,6}$ When the known potential for equipment errors and physiologic patient variations are also considered, it would not be surprising to find differences in systolic and diastolic blood pressure measurements exceeding $\pm 28 \mathrm{mmHg}$ and $\pm 20 \mathrm{mmHg}$, respectively,

*Numbers are derived from four studies and represent the average of their 95 percent confidence limits $(1.96 \times$ standard deviation of the differences between readings). 
Table 1. Factors Confounding the Accuracy of Auscultatory Blood Pressure Measurements

\begin{tabular}{|c|c|c|}
\hline Confounding Factor & Effect(s) and Degree of E.ffect(s) & Remedy \\
\hline $\begin{array}{l}\text { Body posture } \\
\text { Sitting without back support } \\
\text { Standing } \\
\text { Lying (greater difference in pregnancy) }\end{array}$ & $\begin{array}{l}\text { Increased DBP }>\mathrm{SBP}(5-10 \mathrm{~mm} / \mathrm{Ig})^{3} \\
\text { Increased DBP }>\mathrm{SBP}^{3} \\
\text { Decreased DBP }>\mathrm{SBP}^{3}\end{array}$ & $\begin{array}{l}\text { Use standard seated position with back } \\
\text { support }\end{array}$ \\
\hline Arm support - active support (vs passive) ${ }^{4}$ & Increased DBP \& SBP $(4+\mathrm{mmI} \mathrm{Ig})^{+}$ & Use passive support of arm \\
\hline $\begin{array}{l}\text { Arm position }{ }^{+} \\
\text {At level of sternomanubrial junction } \\
\text { At level of xiphoid process }\end{array}$ & $\begin{array}{l}\text { Decrease in DBP \& SBP }(5 \mathrm{mml} / \mathrm{Ig}) \\
\text { Increase in DBP \& SBP }(5 \mathrm{mml} \mathrm{Ig})\end{array}$ & $\begin{array}{l}\text { Use standard arm position at fourtl } \\
\text { intercostal space }\end{array}$ \\
\hline Rate of deflation - too fast ${ }^{1,4}$ & $\begin{array}{l}\text { Underestimation of } \mathrm{SBP}^{3} \\
\text { Overestimation of } \mathrm{DBP}^{3}\end{array}$ & Deflate at $2 \mathrm{~mm} / \mathrm{g} / \mathrm{sec}$ \\
\hline Auscultatory gap - missed & Underestimation of SBP1,3,4 & $\begin{array}{l}\text { 1. Determine point of maximal inflation } \\
\text { by palpation, then inflate to } 30 \mathrm{~mm} I \mathrm{Ig} \\
\text { above this value, }{ }^{4} \text { or } \\
\text { 2. Inflate to } 230 \mathrm{mml} \mathrm{Ig}\end{array}$ \\
\hline $\begin{array}{l}\text { Pressure on head of stethoscope } \\
10 \mathrm{mmI} \mathrm{Ig} \\
\text { Firm pressure }\end{array}$ & $\begin{array}{l}\text { Decreases DBP }\{\mathrm{K}-\mathrm{V}\}\left(10 \mathrm{mmI} \mathrm{Ig}^{3}\right. \\
\text { Sounds may persist down to } 0 \mathrm{mmL} \mathrm{Ig}^{3}\end{array}$ & $\begin{array}{l}\text { Apply only enough pressure to head of } \\
\text { stethoscope to seal against skin }\end{array}$ \\
\hline Use of bell vs diaphragm & $<2 \mathrm{~mm}$ Ig difference ${ }^{3}$ & $\begin{array}{l}\text { Considered insignificant (use either bell } \\
\text { or diaphragm) }\end{array}$ \\
\hline $\begin{array}{l}\text { Size of cuff } \\
\text { Too small } \\
\text { Too large }\end{array}$ & $\begin{array}{l}\text { Overestimation of SBP }(5-9.5 \mathrm{mmH})^{3} \\
\text { Overestimation of DBP }(4-7 \mathrm{mml} \mathrm{gg})^{3} \\
\text { Underestimation of SBP }(0-4 \mathrm{mml} \mathrm{gg})^{3}\end{array}$ & $\begin{array}{l}\text { Consider always using large cuffs, as the } \\
\text { degree of effect if cuff is too large seems } \\
\text { negligible }\end{array}$ \\
\hline No resting period between readings ${ }^{1,4}$ & Inaccurate auscultation of K-I and $\mathrm{K}-\mathrm{V}$ & Allow at least $30 \mathrm{sec}$ between readings \\
\hline Lack of bilateral measurements & $\begin{array}{l}\text { Up to } 10 \mathrm{mmI} I g \text { difference between } \\
\text { arms in } 1 \%-6 \% \text { of patients } \\
\text { 1,3,4 }\end{array}$ & $\begin{array}{l}\text { Take measurements in both arms, and then } \\
\text { follow the arm with the higher pressure } \\
\text { Always document on which arm the reading } \\
\text { is taken }\end{array}$ \\
\hline $\begin{array}{l}\text { Improper selection of Korotkoff sounds } \\
(\mathrm{DBP})^{5}\end{array}$ & Overestimation of DBP $(5-10 \mathrm{mml} \mathrm{Ig})^{3,4}$ & $\begin{array}{l}\text { Make sure that } \mathrm{K}-\mathrm{V} \text { is selected as the DBP } \\
\text { (the very last sound heard) }\end{array}$ \\
\hline $\begin{array}{l}\text { Digit preference (rounding to nearest } \\
5 \text { or } 10 \mathrm{mmI}(\mathrm{Ig})\end{array}$ & $0.5 \mathrm{mmHg}$ inaccuracy & Round to the nearest $2 \mathrm{mmI} \mathrm{Ig}^{4}$ \\
\hline $\begin{array}{l}\text { Equipment errors }{ }^{1,5} \\
\text { Aneroid sphygmomanometers } \\
\text { Mercury column manometers }\end{array}$ & $\begin{array}{l}22 \%-60 \% \text { inaccurate }^{1} \\
2 \%-8 \% \text { inaccurate }^{1}\end{array}$ & Regular inspection, repair, and calibration \\
\hline $\begin{array}{l}\text { Environmental } \\
\text { Cold room }\left(12^{\circ} \mathrm{C} \text { vs } 24^{\circ} \mathrm{C}\right) \\
\text { Talking during measurement }\end{array}$ & $\begin{array}{l}\text { Increase in DBP (up to } 15 \mathrm{mmH} \mathrm{Ig})^{1} \\
\text { Increase in DBP }(8-15 \mathrm{mmH})^{1}\end{array}$ & $\begin{array}{l}\text { Take measurements in warm rooms } \\
\text { No talking when taking reading }\end{array}$ \\
\hline $\begin{array}{l}\text { Pseudohypertension } \\
\text { (from atherosclerosis) })^{3,4}\end{array}$ & $\begin{array}{l}\text { Overestimation of SBP/DBP } \\
\quad(\geq 15 \mathrm{mmH})\end{array}$ & $\begin{array}{l}\text { Clinical suspicion } \\
\text { Osler maneuver (suggestive test) }\end{array}$ \\
\hline Cuff-inflation hypertension ${ }^{4}$ & Overestimation of SBP/DBP & Clinical suspicion (debated) \\
\hline
\end{tabular}

DBP - diastolic blood pressure, SBP - systolic blood pressure, K - Korotkoff.

for the same patient between office visits 5 percent of the time. ${ }^{7,8}$ Just how much of this variation is due solely to practitioner technique is unclear, but one report has estimated that measurements can vary 15 $\mathrm{mmHg}$ or more from basal blood pressure. ${ }^{4}$

Anecdotal concerns regarding the accuracy of nursing blood pressure readings led to a small uncontrolled local study showing highly significant differences between nurse and physician readings for the same patient during the same visit. This current study was performed to better quantify the accuracy of blood pressure readings obtained by nurses in three family practice centers.

\section{Methods}

\section{Setting}

The three PinnacleHealth System family practice residency centers were selected for this study. Centers 1 and 2 are urban practices of 6000 to 7000 patients each. Center 3 is a suburban-rural practice of approximately 15,000 patients. Each center has a physician medical director and a nurse manager. The three centers share one administrator. 
Table 2. Characteristics of Study Variables.

\begin{tabular}{lc}
\hline Characteristic & Number \\
\hline Total sets of blood pressure & 166 \\
readings & \\
$\quad$ Center 1 & 52 \\
$\quad$ Center 2 & 59 \\
$\quad$ Center 3 & 55 \\
Personnel assessed at center 1 & 7 (2 MAs, 2 LPNs, 3 RNs) \\
Personnel assessed at center 2 & 9 (3 MAs, 6 LPNs, 0 RNs) \\
Personnel assessed at center 3 & 10 (3 MAs, 6 LPNs, 1 RN) \\
Obesity* absent & 74 \\
Obesity present & 92 \\
Cardiovascular disease ${ }^{\dagger}$ absent & 108 \\
Cardiovascular disease present & 58 \\
Reading taken in morning & 101 \\
Reading taken after noon & 65 \\
\hline
\end{tabular}

MA - medical assistant, LPN - licensed practical nurse, RN - registered nurse.

"Height and weight were recorded, with ideal body weight $(\mathrm{IBW})=100 \mathrm{lb}+5 \mathrm{lb}$ (height in inches $-60 \mathrm{in}$ ) for women, and $I B W=105 \mathrm{lb}+5 \mathrm{lb}$ (height in inches $-60 \mathrm{in}$ ) for men. Obesity $=$ $\mathrm{IBW}+0.1 \mathrm{IBW}+0.2(\mathrm{IBW}+0.1 \mathrm{IBW})$

+ Cardiovascular disease included history of high blood pressure, diabetes mellitus, coronary artery disease, arteriosclerotic heart disease, atrial fibrillation, congestive heart failure, or arteriosclerotic peripheral vascular disease.

The true subjects of this study were the staff nurses and medical assistants at each center. $\mathrm{Pa}$ tients aged 18 years or older who came to the centers during the days of the study were randomly involved. Evidence of cardiovascular disease, height, and weight (to calculate for obesity) were recorded for each patient, as these factors could make measuring blood pressure technically more difficult. The staff responsible for blood pressure measurements for all patients at each center consisted of a mixture of medical assistants, licensed practical nurses (majority), and registered nurses. Table 2 summarizes pertinent demographics of the staff and patients involved in the study.

\section{Procedures}

This prospective, blinded study evaluated the ability of staff nurses and medical assistants (study nurses) to obtain accurate blood pressure measurements. Blood pressure measurement by an experienced registered nurse (control nurse) with no reported hearing difficulties from a reliable temporary agency was used as the reference standard with which the study nurses' measurements were compared.

The control nurse's technique was developed, after reading the study protocol and supporting literature, ${ }^{1-5,7}$ in three 1.5 -hour morning sessions of blood pressure measurement using modified AHA recommendations for determining blood pressure by sphygmomanometry (Table 3). Blood pressure readings of volunteers were obtained by the control nurse as well as two trainers, and the technique was refined until blood pressure readings regularly agreed to within $\pm 4 \mathrm{mmHg}$. The volunteers were kept in the same room, chair, and position for each set of three readings. One to 2 minutes were allowed between readings, the same blood pressure device was used for each set of readings, and the order of taking measurements was intentionally varied among the three participants. Table 4 includes the precise variability limits attainable with this instruction and practice. The control nurse's technique was thereby certified as correct and her readings considered to represent as close to the basal readings as could be obtained (ie, the reference standard).

During 3-day periods at each center the control nurse rechecked the study nurses' blood pressure readings on a convenience sample of patients. Physiologic factors were limited by (1) keeping the patient in the same room and in the same position for both study nurse and control nurse readings; (2) arranging the flow of information gathering in such a way as to keep 1 to 2 minutes between comparison readings; (3) using the same arm to take both readings; and (4) randomizing (by the throw of a die) the order in which the study nurses and control nurse took their measurements (to neutralize the statistical effects of any phasic or time differences). Equipment factors were eliminated by using the same blood pressure device for comparison readings. In centers 1 and 3 mercury manometers were used; in center 2 aneroid sphygmomanometers were used.

Study nurses were not informed about the purpose of the study other than notification several days before that a "quality improvement blood pressure study" would take place. Neither the control nurse nor the study nurses were aware of each other's readings. In addition to each pair of blood pressure measurements, the control nurse recorded the center, the study nurse's name, the time of day, whether the patient had any cardiovascular diseases (Table 2), and the height and weight of each patient.

Before beginning the main study, all center 1 mercury sphygmomanometers were inspected and 
calibrated, revealing a widespread need for tubing replacement on most of the devices. Because the study protocol dictated the use of the same measurement device for each pair of readings (to nullify equipment errors), further calibration of devices at the other two centers was not considered necessary for the purposes of this study.

\section{Analyses}

The data for training and regular sessions were analyzed using one-way and two-way paired ttests, in addition to one-way analysis of variance (ANOVA). The means of the absolute values of the differences between readings were also calculated, as the practicing clinician would want to know the average variance to expect for each blood pressure reading regardless of whether that variance is greater or lesser than the true reading.

After completion of the study, an analysis of the order in which the blood pressures were taken (ie, whether the control nurse or study nurse took the reading first) showed no significance for diastolic readings and borderline significance for systolic readings $(P=0.049)$. When days 1 through 9 were compared, there were no significant or progressive trends in the data that would suggest failure to implement the study. Poststudy qualitative review with the control nurse confirmed careful attention to following the protocol through the very last day of data collection.

\section{Results}

A total of 166 sets of blood pressure measurements and related data were collected during the main study, and 54 sets of blood pressure measurements were taken during the training sessions (Table 2).

The average systolic and diastolic readings for the sample as a whole obtained by control and study nurses were $126.10 / 78.90 \mathrm{mmHg}$ and $125.58 / 77.42 \mathrm{mmHg}$, respectively. This difference was similar to the mean and the diastolic and systolic readings of $115.11 / 73.11 \mathrm{mmHg}$ and $114.15 / 71.70 \mathrm{mmHg}$, respectively, for control nurse and trainers during the training sessions.

When comparing the individual pairs of readings obtained by the control and study nurses, the absolute difference in diastolic blood pressure readings averaged $4.71 \mathrm{mmHg}$, only $0.71 \mathrm{mmHg}$ greater than the average absolute difference achieved during the training sessions. Interest-
Table 3. American Heart Association Recommendations (Modified) for Determining Blood Pressure

by Sphygmomanometry.

1. The patient's arm should be supported at heart level

2. The patient should be sitting with back support

3. The manometer should be at eye level, easy to read, and deflated to zero before measurement begins

4. The proper size bladder should be selected (when in doubt, select the larger size)

5. The bladder center, located by folding the cuff in half, is aligned with the brachial artery, and the cuff should be aftixed so that its inferior edge lies $2.5-3.0 \mathrm{~cm}$ above the antecubital fossa

6. The point of maximal inflation should be identified by palpating the radial artery and inflating the cuff to $30^{\circ}$ $\mathrm{mmIg}$ above the pressure where the pulse is last palpated. If radial artery cannot be palpated, inflate cuff up to $230 \mathrm{mmII}$

7. The bell or diaphragm of the stethoscope can be used, but must be pressed lightly over the brachial artery

8. The cuff is rapidly inflated to the predetermined point of maximal inflation and then deflated slowly at $2-3$ $\mathrm{mmHg} / \mathrm{sec}$

9. The systolic blood pressure, the point at which at least two consecutive faint tapping beats are heard, is noted, and recorded in even numbers (K-I)

10. For adults the diastolic pressure is taken as the point at which the last muffled sound is heard (K-V). After 10 $20 \mathrm{mmHg}$ of silence, the cuff can be rapidly deflated to zero

11. Record the systolic and diastolic pressures as well as the cuff size, the patient's position, and the arm used in the measurement

12. No talking should occur between technician and patient during the measurement of the blood pressure ${ }^{1}$

13. Repeat measurements should be delayed by $1-2$ minutes to allow time for blood to drain from the arm

Modified from Bailey and Bauer, ${ }^{1}$ Campbell et $a^{1,2}$ Baker and Ende, ${ }^{3}$ Campbell et al, ${ }^{4}$ and Frohilch. ${ }^{9}$

ingly, one-way ANOVA among centers was significant $(P=0.002)$ with analysis of the actual diastolic differences, identifying a highly significant 3.78-mmHg negative difference at center 3 (lower readings by the center study nurses). Analysis of the performance of individual study nurses in all centers approached significance for diastolic measurement (ANOVA $=1.56, P=0.057$ ), again with nurses from center 3 largely responsible. In addition, only in center 3 did afternoon readings show significantly less accuracy (difference $=3.77$ $\mathrm{mmHg}, P=0.025$ ). These findings together suggested a systemic error at center 3 in obtaining accurate diastolic pressure readings that resulted in a skewing of the data mostly in one direction. Similar skews in blood pressure measurements, however, were not found at any other center for either diastolic or systolic readings.

When comparing the individual pairs of read- 
Table 4. Differences in Blood Pressure Readings: Main Study and Training Sessions.

\begin{tabular}{|c|c|c|c|c|c|c|c|c|}
\hline \multirow[b]{2}{*}{ Variable $^{*}$} & \multicolumn{3}{|c|}{ Main Study } & \multicolumn{2}{|c|}{ Training Sessions } & \multirow[b]{2}{*}{ No. } & \multirow[b]{2}{*}{$\begin{array}{c}\text { Mean Error } \\
\text { Potentially } \\
\text { Correctable } \\
\text { by Trainings } \\
\text { (mmHg) }\end{array}$} & \multirow[b]{2}{*}{$\begin{array}{c}\text { Excess } \\
\text { Potentially } \\
\text { Correctable } \\
\text { by Training } \\
\text { (mmHg) }\end{array}$} \\
\hline & $\begin{array}{l}\text { Mean of } \\
\text { Absolute } \\
\text { Differences } \\
\text { (mmHg) }\end{array}$ & $\begin{array}{c}\text { Variability }{ }^{\ddagger} \\
\text { (SD) } \\
(\mathrm{mmHg})\end{array}$ & No. & $\begin{array}{c}\text { Mean of } \\
\text { Absolute } \\
\text { Differences } \\
\text { (mmHg) }\end{array}$ & $\begin{array}{c}\text { Variability }{ }^{\ddagger} \\
\text { (SD) } \\
(\mathrm{mmHg})\end{array}$ & & & \\
\hline \multicolumn{9}{|l|}{ Systolic BP } \\
\hline Total & $6.21(0.51)$ & 8.53 & 166 & 4.37 & 5.81 & 54 & 1.84 & \pm 2.72 \\
\hline Center 1 & $6.27(0.88)$ & 9.94 & 52 & & & & & \\
\hline Center 2 & $5.97(0.27)$ & 7.75 & 59 & & & & & \\
\hline Center 3 & $6.42(0.42)$ & 8.04 & 55 & & & & & \\
\hline \multicolumn{9}{|l|}{ Diastolic BP } \\
\hline Total & $4.71(1.49)$ & 6.21 & 166 & 4.00 & 5.74 & 54 & 0.71 & \pm 0.47 \\
\hline Center 1 & $4.54(1.04)$ & 6.10 & 52 & & & & & \\
\hline Center 2 & $4.10(-0.24)$ & 5.74 & 59 & & & & & \\
\hline Center 3 & $5.53(3.78)$ & 6.20 & 55 & & & & & \\
\hline
\end{tabular}

BP - blood pressure.

*All differences were calculated by subtracting the study nurse or trainer readings from the control nurse reading.

tNumbers in parentheses represent the means of the actual differences (not absolute), and demonstrates that most of the diastolic error at center \#3 was in one direction (those in italics reached statistical significance).

₹Statistically derived from analyses of the actual (not absolute) systolic and diastolic differences. Note: standard deviations cannot be derived from nonnormally distributed data, such as absolute values.

$\$$ Calculation $=$ mean of absolute differences from main study minus mean of absolute differences for training sessions.

"Calculation = variability from main study minus variability from training sessions.

ings obtained by control and study nurses, the absolute difference in systolic blood pressure readings averaged $6.21 \mathrm{mmHg}$ : this time $1.84 \mathrm{mmHg}$ greater than the average absolute difference achieved during the training sessions for systolic measurement. Though unidirectional skewing of significance was absent for systolic readings between centers, quite a bit of variability existed: $2.72 \mathrm{mmHg}$ greater than that observed during the training sessions. Interestingly, systolic blood pressure readings in obese patients were more accurate (mean of actual differences for obese patients $-0.70 \mathrm{mmHg}$, and mean for nonobese patients $2.01 \mathrm{mmHg}, P=0.042$ ), traceable only to center 1 .

Table 4 includes the main study results regarding systolic and diastolic blood pressure readings.

\section{Discussion}

In this study average absolute differences in systolic and diastolic blood pressure readings were $6.21 \mathrm{mmHg}$ and $4.71 \mathrm{mmHg}$ between control and study nurses, respectively. If the training session data are assumed to represent the most accurate technique that can be achieved, then average absolute blood pressure differences potentially correctable by training were only $1.84 \mathrm{mmHg}$ sys- tolic and $0.71 \mathrm{mmHg}$ diastolic (Table 4). Such small variations from the reference standard reflect favorably on the ability of the staff nurses to follow accepted blood pressure measurement techniques in the office setting.

Analysis of the average actual differences (positive or negative) revealed an error of almost 4 $\mathrm{mmHg}$ in one direction for diastolic readings at center 3 , suggesting poor implementation of some aspect(s) of accepted technique among staff at that center. Consistent with this theory was the finding that center 3 alone had less agreement between pairs of diastolic readings in the afternoons (as compared with the mornings), presumably from less careful technique toward the end of a full day's work. When data for individual center 3 nurses were analyzed, 4 of 10 study nurses had readings consistently lower than those of the control nurse; 3 of these 4 nurses took the blood pressure measurements almost exclusively in the afternoons. Patient factors, such as obesity or cardiovascular diseases, did not help explain the diastolic differences found at center 3 .

Finally, variation in systolic readings (as represented by the standard deviation of the actual differences in systolic blood pressure readings) was higher in all three centers $( \pm 8.53 \mathrm{mmHg})$ than 
during the training sessions for the control nurse $( \pm 5.81 \mathrm{mmHg}$ ). Once again, if the training sessions data are assumed to represent the most accurate technique that can be achieved, then systolic variability potentially correctable by training becomes just $\pm 2.72 \mathrm{mmHg}$, a very tolerable number, indeed.

Previous studies have reported differences in systolic pressure measurement of $4 \mathrm{mmHg}$ or more to be to the following variables: rate of deflation, ${ }^{3}$ missing an auscultatory gap, ${ }^{1,3,4}$ terminal digit preferences, 4,5 and arm support and position. ${ }^{4}$ Differences of 4 to $15 \mathrm{mmHg}$ and greater in diastolic pressure measurement have been previously documented for rate of deflation, ${ }^{3}$ pressure on the head of the stethoscope, ${ }^{3}$ improper selection of Korotkoff sounds, ${ }^{3-5}$ terminal digit preference, ${ }^{4,5}$ and talking during measurement. ${ }^{1}$ Taken as a group, as in this study, these variables could produce large discrepancies in comparative readings. The relatively small magnitude of the differences found in the current study suggests, however, that technique errors by the study nurses either were largely absent or, if present, were aligned in opposite directions.

Of course, in the practicing physician's office, currently controlled factors such as body posture, blood pressure cuff size, using different arms for measurement, using different noncalibrated devices, room temperature, and physiologic variations that occur with time would necessarily contribute to further inaccuracies in obtaining useful and comparable readings. Having only 1 or 2 nurses taking measurements, rather than the many nurses in the study centers, would amplify any existing errors in following established protocol, just as office physicians, when rechecking blood pressures, would be likely to regularly reproduce errors in their own techniques. Indeed, the pilot study for this project documented an average actual difference of $10 \mathrm{mmHg}$ in both systolic and diastolic readings during the same visits on the same patients. Only 1 physician and a few nursing assistants were involved in the study, which strongly suggests that the same error or errors were affecting the readings at each measurement. Admittedly, the current study did not attempt to assess all factors that could affect the accuracy of auscultatory blood pressure measurement.

In addition, direct comparison of main study with training session data must be made cautiously because of the following differences in the two parts of the study: (1) there were fewer volunteers (7) for the training sessions than there were patients whose blood pressures were measured, and each volunteer stayed for more than one pair of measurements, whereas there was a different patient for each pair of measurements in the main study; (2) the averages of blood pressure readings for the training data were lower than those for the main study; (3) the training session data were collected only in the morning, whereas the main study data were collected during both mornings and afternoons; (4) the control nurse was a registered nurse, whereas the study nurses were mostly licensed practical nurses and medical assistants; and (5) this study did not involve an additional phase during which all nurses were trained and then reassessed.

Finally, the current study, which looked at how much inaccuracy in blood pressure readings at the family practice centers was due to technique factors, highlights several important issues for the practicing physician. First, questioning and then studying basic practice procedures are the only ways to find out what is really happening in the office setting. Second, relying on a single blood pressure reading to make any important clinical decision is inadvisable, because so many variables can affect the accuracy of that measurement. Third, even if all possible variables are addressed, the minimum potential error in systolic or diastolic auscultatory blood pressure measurement between different observers is $4 \mathrm{mmHg}$ (based upon the results of the training sessions). Fourth, such factors as equipment calibration, cuff size, bilateral readings, etc, must be addressed if measurements in the office are to possess any validity for comparison with time (Table 1).

Fifth, regular (re)training office nursing personnel in the proper measurement of office blood pressures should help limit inconsistencies. Sixth, physicians themselves must remember to use proper technique when rechecking their patients' blood pressures. Seventh, multiple readings with time should help limit the clinical consequences of individual erroneous measurements, a concept that is already incorporated into the initial diagnostic criteria for hypertension.

Because of many of the above issues, some researchers have assessed 24-hour ambulatory blood pressure monitoring, and initial studies have 
shown better correlation with left ventricular wall thickness by echocardiography than with single or multiple visit office measurements. ${ }^{10,11}$ Nevertheless, this 24-hour method is not supported by long-term and large-scale epidemiologic studies similar to those proving the benefit of diagnosing and treating hypertension based upon casual office readings. ${ }^{12}$ More importantly, recent research supports the validity of continued use of office blood pressure measurements rather than 24-hour ambulatory monitoring in the diagnosis and treatment of hypertension. ${ }^{13}$ It appears that the accurate measurement of blood pressure in the office setting will remain central to the optimal treatment of patients.

Sandy Koch, RN, helped ensure that the study was properly implemented in the family practice centers and participated in the training sessions. Allen Shaughnessy, PharmD, and Howard Robertson, EdD, provided expert advice in study design and data interpretation.

\section{References}

1. Bailey $\mathrm{RH}$, Bauer JH. A review of common errors in the indirect measurement of blood pressure. Sphygmomanometry. Arch Intern Med 1993;153:2741-8.

2. Campbell NR, McKay DW, Chockalingam A, Fodor JG. Errors in assessment of blood pressure: patient factors. Can J Public Health 1994;85 (Suppl 2):S12-7.

3. Baker RH, Ende J. Confounders of auscultatory blood pressure measurement. J Gen Intern Med 1995;10:223-31.
4. Campbell NR, McKay DW, Chockalingam A, Fodor JG. Errors in assessment of blood pressure: blood pressure measuring technique. Can J Pubic Health 1994;85(Suppl 2):S18-21.

5. Grim CM, Grim CE. A curriculum for the training and certification of blood pressure measurement for health care providers. Can J Cardiol 1995;11(Suppl $\mathrm{H}): 38 \mathrm{H}-42 \mathrm{H}$.

6. Bogan B, Kritzer S, Deane D. Nursing student compliance to standards for blood pressure measurement. J Nurs Educ 1993;32:90-2.

7. Bottini PB, Carr AA. Variability in indirect blood pressure measurement. Arch Intern Med 1994;154:1536.

8. Bottini PB, Carr AA, Rhoades RB, Prisant LM. Variability of indirect methods used to determine blood pressure: office vs mean 24-hour automated blood pressures. Arch Intern Med 1992;152:139-44.

9. Frohlich ED. Blood pressure measurement. Can J Cardiol 1995;11(Suppl H):35H-37H.

10. Prisant LM, Carr AA, Bottini PB, Thompson WO, Rhoades RB. Repeatability of automated ambulatory blood pressure measurements. J Fam Pract 1992;34: 569-74.

11. Prisant LM, Carr AA. Ambulatory blood pressure monitoring and echocardiographic left ventricular wall thickness and mass. Am J Hypertens 1990;3: 81-9.

12. Stokes J 3rd, Kannel WB, Wolf PA, D'Agnostino RB, Cupples LA. Blood pressure as a risk factor for cardiovascular disease. The Framingham Study - 30 years of follow-up. Hypertension 1989;13(5 Suppl): I13-8.

13. Palatini P, Penzo M, Canali C, Dorigatti F, Pessina A. Interactive action of the white-coat effect and the blood pressure levels on cardiovascular complications in hypertension. Am J Med 1997; 103:208-16. 